\title{
Including inputs and control within equation-free architectures for complex systems
}

\author{
Joshua L. Proctor ${ }^{1, a}$, Steven L. Brunton ${ }^{2}$, and J. Nathan Kutz ${ }^{3}$ \\ 1 Institute for Disease Modeling, Bellevue, WA 98004, USA \\ 2 Mechanical Engineering, University of Washington, Seattle, WA 98195, USA \\ 3 Department of Applied Mathematics, University of Washington, Seattle, WA 98195, USA
}

Received 19 February 2016 / Received in final form 28 June 2016

Published online 22 November 2016

\begin{abstract}
The increasing ubiquity of complex systems that require control is a challenge for existing methodologies in characterization and controller design when the system is high-dimensional, nonlinear, and without physics-based governing equations. We review standard model reduction techniques such as Proper Orthogonal Decomposition (POD) with Galerkin projection and Balanced POD (BPOD). Further, we discuss the link between these equation-based methods and recently developed equation-free methods such as the Dynamic Mode Decomposition and Koopman operator theory. These data-driven methods can mitigate the challenge of not having a well-characterized set of governing equations. We illustrate that this equation-free approach that is being applied to measurement data from complex systems can be extended to include inputs and control. Three specific research examples are presented that extend current equation-free architectures toward the characterization and control of complex systems. These examples motivate a potentially revolutionary shift in the characterization of complex systems and subsequent design of objective-based controllers for data-driven models.
\end{abstract}

\section{Introduction}

The characterization and control of complex systems permeate classic physical, biological, and engineering sciences and enable modern applications such as the eradication of Poliomyelitis, control of internet traffic, optimizing energy infrastructures, and social media advertising. The rapidly expanding capabilities in computing power, data storage, and data transfer rates have generated enormous data sets, novel experiments, and offered inspiration to control complex systems in real-time. Adapting traditional mathematical and engineering methods to these high-dimensional, nonlinear systems has presented substantial challenges. Moreover, the design of controllers and the characterization of these complex systems remain an open-challenge requiring

\footnotetext{
${ }^{a}$ e-mail: joproctor@intven.com
} 
the development of new quantitative methods. We review the traditional approaches to data-driven analysis while highlighting a number of recent methodological advances toward the control of complex systems.

Despite the dimensionality and complexity, it is often possible to find a lowdimensional model to represent the input-output characteristics of the system. This observation is well-known in the field of dynamical systems, specifically within the model reduction community. Identifying the correct, qualitative types of solutions from complex systems is the primary objective of normal forms [1]. The analysis of solutions from higher dimensional systems such as those found in fluid dynamics led to model reduction techniques such as the Proper Orthogonal Decomposition (POD) [2-8] which have been extended to numerous other fields such as animal locomotion $[9,10]$, vibrational analysis [11-13], and damage analysis [14,15]. Combining POD with known governing equations via Galerkin projections produces ReducedOrder Models (ROMs) that have been used to analyze fluid flows $[3,4,6,16]$, optical systems [17-19], and fluid flows with control [20-22]. Recent advances have illustrated how POD/Galerkin projections can be extended to more efficiently handle the model reduction component involving the nonlinearities of partial differential equations [23-25].

For engineered systems, discovering advantageous dynamical regimes is important for objective-based control. The rich literature of system identification within the control theoretic community has taken a parallel perspective to POD. In fact, the Singular Value Decomposition (SVD), which is the dimensionality-reduction technique utilized in $\mathrm{POD}$, is also prominently used to construct low-dimensional subspaces where controller design is computationally tractable [6,26-33]. Balanced truncation is a foundational technique that produces ROMs that are constructed to balance input-output characteristics such as controllability and observability [26]. Generalizations of this method, such as Balanced Proper Orthogonal Decomposition (BPOD), utilize the SVD on high-dimensional measurement data to produce balanced ROMs of complex systems, but the method still requires a difficult linear adjoint calculation [29,34-36]. System identification methods do not rely on this adjoint calculation and were developed to aid in the discovery of input-output models for systems with control [27,37-41]. Further, these system identification methods can be considered equation-free since they do not rely on a set of governing equations such as with POD/Galerkin projection and BPOD. A fundamentally important observation by $\mathrm{Ma}$ et al. demonstrated that the Eigensystem Realization Algorithm (ERA), a system identification method, reproduces balanced input-output models similar to BPOD, thus linking equation-based and equation-free methods [42]. We briefly review POD and BPOD in the Background section because of the historical context and the direct relationship to current equation-free methods. Other system identification methods called subspace identification methods bypass the identification of Markov parameters to produce input-output models from measurement data [43-46].

Dynamic Mode Decomposition (DMD) is a data-driven methods that operates on snapshot measurement only and is considered an equation-free architecture [47-52]. Early success for DMD has been achieved on fluid dynamics problems [52-57], and has been subsequently extended to epidemiology [58], neuroscience [59], and foreground/background separation in video streams [60]. DMD has been shown to be connected to system identification methods such as ERA [51] and equation-based methods such as BPOD [42]. ERA has been predominantly used on systems where the number of measurements is assumed to be low and the system linear whereas DMD excels on high-dimensional measurement data from complex, nonlinear systems [61]. The architecture also lends itself to enabling extensions that take advantage of innovative sampling strategies in space and time [57,62-64], multi-resolution/ 
multi-scale phenomenon [65], de-noising [66,67], and data fusion [68], extended and kernel DMD $[69,70]$. The method was also extended to handle data from complex systems with inputs [61]. DMD has been shown to be a linear, finite-dimensional approximation of a powerful spectral analysis method for nonlinear dynamical systems called Koopman operator theory $[48,71,72]$. Koopman operator theory has been rapidly permeating a number of scientific and engineering fields [73,74]. Further, the Koopman architecture is being extended to investigate systems with input-output characteristics $[75,76]$.

Due to the ubiquitous use of the SVD for equation-based methods such as POD and BPOD as well as equation-free methods such as DMD and Koopman, we begin the background section with a mathematical description of the SVD. We then describe traditional model-based reduction techniques for dynamical systems with POD/Galerkin projection and input-output systems with BPOD. These equationbased methods are fundamentally linked to equation-free methods such as the recently developed DMD $[42,51]$. The background also provides a review of both DMD and Koopman operator theory. The subsequent sections highlight, in more specific detail, three recent developments that extend these equation-free methods to allow for input-output systems.

\section{Background and method implementation}

We begin with a standard linear algebra method utilized in model-based dimensionality reduction and equation-free techniques: the SVD. This technique is essential to the formulation of POD/Galerkin models for dynamical systems, BPOD for input-output systems, and DMD for time-series data. We begin the background section by describing traditional model-based methods such as POD/Galerkin projections and BPOD. Despite the fundamental difference between equation-based methods such as BPOD and equation-free methods such as DMD, these techniques are theoretically connected via the Eigensystem Realization Algorithm (ERA) and have been shown to produce similar balanced models [42,51]. We follow the description of BPOD with a review of DMD and it's theoretical connections to Koopman operator theory, a data-driven perspective on analyzing nonlinear dynamics. We present the historical context of these methods as well as the mathematical formulation.

\subsection{The singular value decomposition}

The SVD is a model reduction technique that is essential to POD and DMD. The SVD has a rich history in data analysis and is being used in a diverse set of fields such as statistics, biology, computer science, and applied engineering [77-79]. The SVD is a factorization of a data matrix $\mathbf{X}$ given by:

$$
\mathbf{X}=\mathbf{U} \boldsymbol{\Sigma} \mathbf{V}^{*}=\left[\begin{array}{ll}
\tilde{\mathbf{U}} & \tilde{\mathbf{U}}_{\mathrm{rem}}
\end{array}\right]\left[\begin{array}{cc}
\tilde{\boldsymbol{\Sigma}} & 0 \\
0 & \boldsymbol{\Sigma}_{\mathrm{rem}}
\end{array}\right]\left[\begin{array}{c}
\tilde{\mathbf{V}}^{*} \\
\tilde{\mathbf{V}}_{\mathrm{rem}}^{*}
\end{array}\right] \approx \tilde{\mathbf{U}} \tilde{\boldsymbol{\Sigma}} \tilde{\mathbf{V}}^{*}
$$

where $\mathbf{X} \in \mathbb{R}^{n \times m}$ is the data matrix with $n$ features and $m$ samples, $\mathbf{U} \in \mathbb{R}^{n \times n}$ are the left singular vectors, $\boldsymbol{\Sigma} \in \mathbb{R}^{n \times m}$ are the singular values ranked by magnitude, and $\mathbf{V} \in \mathbb{R}^{m \times m}$ are the right singular vectors. Choosing a truncation value $r$, where $r \ll n$, allows for a low-rank approximation of $\mathbf{X} \approx \tilde{\mathbf{U}} \tilde{\boldsymbol{\Sigma}} \tilde{\mathbf{V}}^{*}$ with matrices $\tilde{\mathbf{U}} \in \mathbb{R}^{n \times r}$, $\tilde{\boldsymbol{\Sigma}} \in \mathbb{R}^{r \times r}$, and $\tilde{\mathbf{V}}^{*} \in \mathbb{R}^{r \times m}$ with rank at most $r[16,79,80]$. If the low-rank approximation is exact, the matrix $\boldsymbol{\Sigma}_{\text {rem }}$ will contain all zeros. 

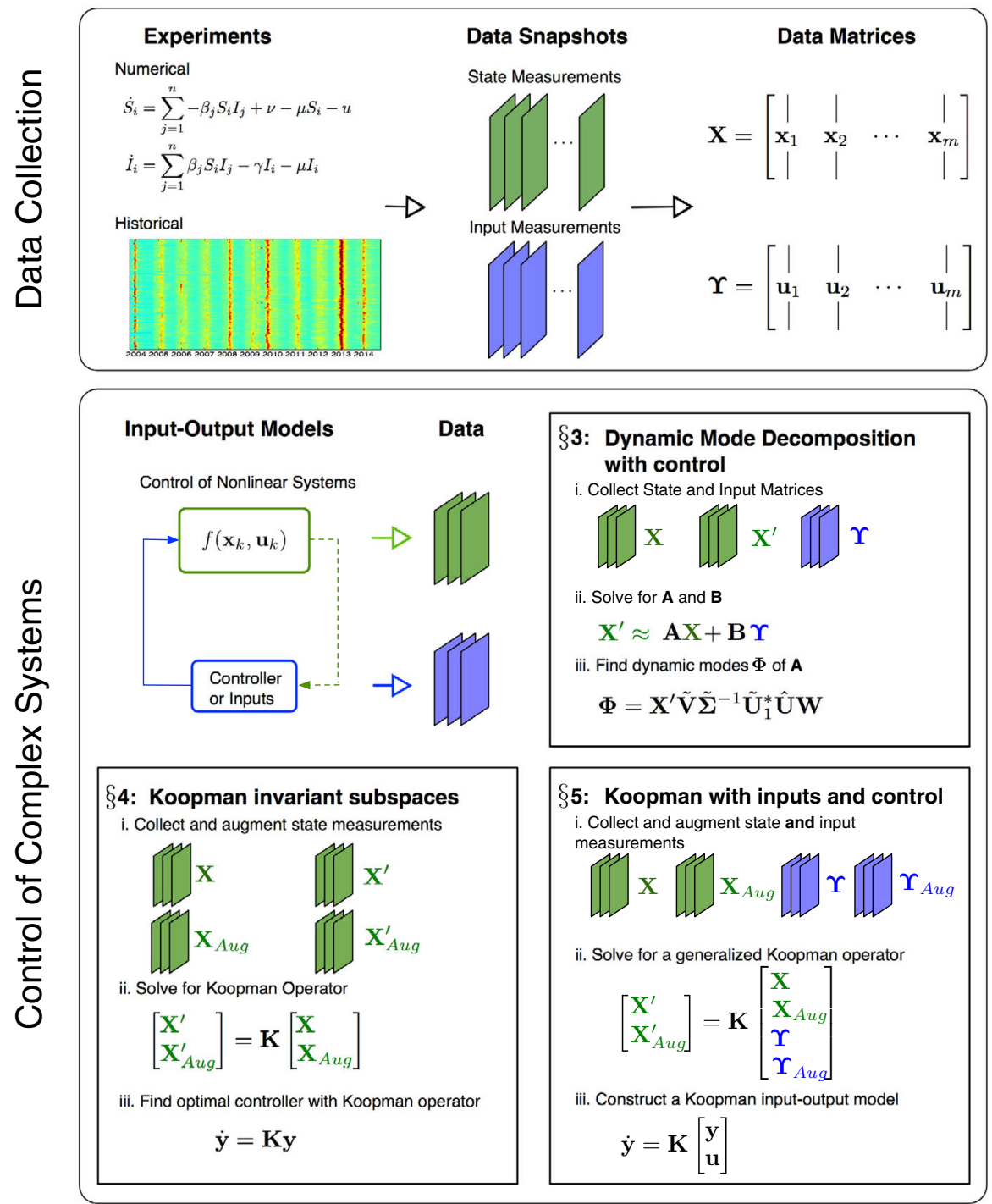

Fig. 1. The top panel illustrates the data collection aspect of equation-free methods. The data can come from experiments, numerical simulations, or historical records. In this case, the historical records illustrate google flu trends in the United States with the location on the $\mathrm{y}$-axis and time on the $\mathrm{x}$-axis. The bottom panel presents the three recently developed perspectives on characterizing nonlinear input-output systems using equation-free techniques.

Choosing the appropriate truncation value $r$ has a long and extensive history. There are a number of statistical methods and heuristics that have been used for truncation criteria [81]. The Eckart-Young theorem is one of the most commonly utilized methods for choosing $r$ [77-79]. The theorem states that the best approximation of $\mathbf{X}$ with $k$ modes can be found by retaining the $k$ largest singular values and respective modes. Recent theoretical developments attempt to optimally identify $r$ when $\mathbf{X}$ may have additive noise $[82,83]$. 


\subsection{The proper orthogonal decomposition and galerkin projection}

POD and Galerkin projections is a model reduction technique that has been integral in the analysis of dynamical systems. The goal of POD is to discover a low-dimensional subspace on which solutions of complex systems evolve. POD is known in other fields as Principal Components Analysis (PCA) [84] (with the mean subtracted out), the Hotelling transform [85], Empirical Orthogonal Functions (EOF) [86,87], and/or the Karhunen-Loéve (KL) decomposition [88]. The method has been utilized in a large and diverse set of scientific and engineering applications [2-13].

POD combined with Galerkin projection can also be used to construct ROMs. If a low-rank approximation of the subspace is available, governing equations can be projected on to the POD-discovered subspace [3,6]. This perspective is inherently equation-based requiring knowledge of the governing equations in order to perform the model reduction procedure. These ROMs have been successfully applied to a number of nonlinear fluid dynamics problems [16,89-93] including those with inputs and control [20-22]. A recent development, Discrete Empirical Interpolation Method (DEIM), has notably extended POD and Galerkin projections to more efficiently handle general nonlinear terms in the vector field [24,25], which is a discrete version of [23]. In this subsection, we describe the basic methodological foundation of POD and POD with Galerkin projections applied to complex systems. A description of BPOD follows this subsection describing model reduction for input-output models.

\subsubsection{Implementation}

POD uses measurement data from numerical models, experiments, or historical records to discover a low-dimensional subspace. POD produces a projection $P$ that takes the state $\mathbf{x}(t)$ from the high-dimensional phase space and projects to a low-dimensional subspace. We present the formulation of POD in the context of continuous variables such that $\mathbf{x}(t) \in \mathbb{R}^{n}$ for $t \in[0, T]$. The error between the full-state trajectories and the trajectories on the low-dimensional subspace is defined by:

$$
\int_{0}^{T}\|\mathbf{x}(t)-P \mathbf{x}(t)\|_{2}^{2} d t .
$$

The projection $P$ can be solved for by defining the following representation of the data:

$$
R=\int_{0}^{T} \mathbf{x}(t) \mathbf{x}(t)^{*} d t
$$

where $^{*}$ is the complex-conjugate transpose. Solving the eigenvalue problem for $R$ :

$$
R \mathbf{u}_{k}=\lambda_{k} \mathbf{u}_{k}
$$

provides a subspace spanned by the eigenvectors $\mathbf{u}_{k}$ also known as the POD modes of the data matrix $R$. Using the eigenvectors $\mathbf{u}_{k}$ and choosing a truncation parameter $r$, the projection $P$ can be constructed:

$$
P=\sum_{k=1}^{r} \mathbf{u}_{k} \mathbf{u}_{k}^{*}
$$


Data often comes discretized in time such that $\mathbf{x}\left(t_{j}\right)=\mathbf{x}_{j}$ for $j=1,2, \ldots, m$. The method of snapshots utilizes the SVD to efficiently compute POD modes from data matrices when $n \gg m$.

\section{Method of Snapshots}

The discretized data $\mathbf{x}_{j}$, also called snapshots, can be concatenated in to a data matrix:

$$
\mathbf{X}=\left[\begin{array}{cccc}
\mid & \mid & & \mid \\
\mathbf{x}_{1} & \mathbf{x}_{2} & \ldots & \mathbf{x}_{m} \\
\mid & \mid & & \mid
\end{array}\right]
$$

The discretized description of $\mathbf{R}$ from (3) is $\mathbf{R}=\mathbf{X X}^{*}$, which is size $n \times n$. The method of snapshots refers to solving a related, but computationally smaller problem involving $\tilde{\mathbf{R}}=\mathbf{X}^{*} \mathbf{X}$, which is $m \times m$ [94]. The eigenvalue problem for $\tilde{\mathbf{R}}$ is

$$
\tilde{\mathbf{R}} \mathbf{v}_{k}=\lambda_{k} \mathbf{v}_{k}
$$

where $\mathbf{v}_{k} \in \mathbb{R}^{m}$. The nonzero eigenvalues for $\mathbf{R}$ and $\tilde{\mathbf{R}}$ are equivalent and the eigenvectors are related by:

$$
\mathbf{U}=\mathbf{X V} \Lambda^{-1 / 2}
$$

where $\mathbf{u}_{k}$ and $\mathbf{v}_{k}$ form the singular vectors of $\mathbf{U}$ and $\mathbf{V}$ respectively. These results follow from the fact that $\mathbf{X}^{*} \mathbf{X V}=\mathbf{V} \boldsymbol{\Sigma}^{2}$ and $\mathbf{X} \mathbf{X}^{*} \mathbf{U}=\mathbf{U} \boldsymbol{\Sigma}^{2}$.

\section{Galerkin Projection}

The POD/Galerkin method involves using the POD modes $\mathbf{U}$ of the data matrix $\mathbf{X}$ to project a nonlinear dynamical system onto the low-rank subspace. Given a nonlinear dynamical system

$$
\dot{\mathbf{x}}=f(\mathbf{x})
$$

the singular vectors $\mathbf{U}$ can be used to project the state $\mathbf{x}$ and the vector field onto the low-dimensional subspace

$$
\dot{\tilde{\mathbf{x}}}=\mathbf{U}^{*} f(\mathbf{U} \tilde{\mathbf{x}}),
$$

where the dynamical system is now defined on a state $\tilde{\mathbf{x}}$. The ROM can then be used to investigate bifurcation parameters and design controllers. However, POD/Galerkin expansions have issues with input-output systems [4].

\subsection{Balanced proper orthogonal decomposition}

The POD methodology has been quite successful in the analysis of dynamical systems, but has been problematic for input-output systems $[4,5,7]$. Choosing the truncation value $r$ by the energy of singular values has been shown to incorrectly identify input-output characteristics [36]. BPOD combines POD and balanced truncation, a classic method from the control theoretic community that balances observability and controllability [26]. Related developments involving Hankel-norm approximations also propose to balance these input-output characteristics [95-97]. Balanced truncation is a coordinate transformation that finds states that are both strongly controllable and 
observable [26]. A balanced realization truncates weakly observable and controllable states and constructs a ROM of an input-output system.

Balanced truncation for complex systems with large number of states can be computationally expensive requiring solving Lyapunov equations with dimensions of the state $n \times n$. Data-driven methods involving POD were proposed to bypass this computational difficulty for nonlinear systems [28,34]. For large numbers of outputs, the snap-shot based methods require a large number of adjoint simulations. BPOD is a computationally tractable method that reduces the number of adjoint simulations and approximates balanced truncation $[29,35]$. BPOD has been successfully applied in a number of fluid control systems [36,98-103] and wind farm control systems [104].

\subsubsection{Implementation}

Consider a stable linear input-output system

$$
\begin{gathered}
\dot{\mathbf{x}}=\mathbf{A x}+\mathbf{B u} \\
\mathbf{y}=\mathbf{C x}
\end{gathered}
$$

where $\mathbf{u} \in \mathbb{R}^{l}$ is the vector of inputs, and $\mathbf{y} \in \mathbb{R}^{z}$ is the output vector. Balanced truncation is a control theoretic model reduction technique that balances controllability and observability [26]. The objective is to construct a coordinate transformation which diagonalizes the controllability and observability Gramians, defined by

$$
W_{c}=\int_{0}^{\infty} e^{\mathbf{A} t} \mathbf{B B}^{*} e^{\mathbf{A}^{*} t} d t, \quad W_{o}=\int_{0}^{\infty} e^{\mathbf{A}^{*} t} \mathbf{C}^{*} \mathbf{C} e^{\mathbf{A} t} d t
$$

where ${ }^{*}$ indicates the corresponding linear-adjoint system:

$$
\dot{\mathbf{z}}=\mathbf{A}^{*} \mathbf{z}+\mathbf{C}^{*} \mathbf{v} .
$$

For BPOD, the method calls for collecting data through impulse responses of (11) and the adjoint system (13). Note that data is being collected from both the direct and adjoint systems. The impulse response data from (11) is collected into a data matrix X. The impulse response data from (13) is collected into Y. The data matrices are constructed similarly to the discrete-time version of POD and method of snapshots [94]. The Gramians can be approximated using the impulse response data matrices $\mathbf{X}$ and $\mathbf{Y}$ :

$$
W_{c} \approx \mathbf{X X}^{*}, \quad W_{o} \approx \mathbf{Y Y}^{*},
$$

which are called empirical Gramians [34]. The goal of BPOD is to find a transformation that balances these empirical Gramaians. The method of snapshots helps find a transformation without computing the Gramians themselves. The SVD is used to discover the balancing transformation

$$
\mathbf{Y}^{*} \mathbf{X}=\mathbf{U}_{B} \boldsymbol{\Sigma}_{B} \mathbf{V}_{B}^{*}
$$

from which the transformation $\boldsymbol{\Phi}$ and $\boldsymbol{\Psi}$ are computed by

$$
\mathbf{\Phi}=\mathbf{X} \mathbf{V}_{B} \boldsymbol{\Sigma}_{B}^{-1 / 2}, \quad \boldsymbol{\Psi}=\mathbf{Y} \mathbf{U}_{B} \boldsymbol{\Sigma}_{B}^{-1 / 2} .
$$

The balancing modes $\boldsymbol{\Phi}$ and the adjoint modes $\boldsymbol{\Psi}$ are biorthogonal. The singular values of $\boldsymbol{\Sigma}_{B}$ are known as Hankel singular values. A truncation can be performed 
on the Hankel singular values similar to SVD which helps produce a ROM of the input-output system. A number of large-scale systems, including fluid dynamics face another computational challenge around simulating the adjoint. Each output requires one simulation, resulting in a significant number of simulations when the number of output measurements is large. A projection on the output is performed to alleviate this challenge resulting in the decrease of the effective number of simulations required. A POD projection $\mathbf{P}$ from the impulse-state responses minimizes the error between the original input-output transfer function and the output-projected transfer function [29]. A truncated output projection $\mathbf{P}_{r}$ and a truncated set of biorthogonal modes $\boldsymbol{\Phi}_{r}$ and $\boldsymbol{\Psi}_{r}$ can be used to construct a balanced reduced order model

$$
\dot{\tilde{\mathbf{x}}}=\boldsymbol{\Psi}_{r}^{*} \mathbf{A} \boldsymbol{\Phi}_{r} \tilde{\mathbf{x}}+\boldsymbol{\Psi}_{r}^{*} \mathbf{B u}
$$

$$
\mathbf{y}=\mathbf{P}_{r}^{*} \mathbf{C} \phi_{r} \mathbf{x}
$$

which both balances the input-output characteristics of controllability and observability and is computationally feasible. Note that the formulation of BPOD presented is from [29]. This formulation differs from an earlier version of BPOD [35] in which the empirical Gramians are separately reduced and then balanced.

\subsection{The dynamic mode decomposition}

DMD is a data-driven, equation-free methodology that discovers coherent spatialtemporal modes from measurement data collected from complex systems [47-49]. These spatial-temporal patterns, called dynamic modes and their associated temporal frequencies offer diagnostic insight into the dynamic behavior of a complex system. The dynamic modes are constructed under the fundamental assumption that snapshots of measurement data are connected by the flow of a dynamical system $[48,49]$. This assumption leads to the discovery of modes that are more dynamically relevant than those produced by POD; see [105] for a enlightening comparison between DMD and POD for fluids example of a lid-driven cylindrical cavity. Other equationfree techniques are also being developed that are trying to find macroscopic level equations that describe the evolution of a set of microscopic processes without the need for POD or Galerkin projections [92,106,107]. DMD has also been rigorously connected to Koopman operator theory, which focuses on the analysis of nonlinear vector fields $[48,74]$.

The connection to the analysis of nonlinear systems, the requirement of only measurement data, and the computational tractability of the algorithm has led to a growing number investigations with DMD on a diverse set of engineering and scientific applications. DMD has been developed and applied extensively in the fluid dynamics community where numerically solving a set of complex governing equations for the purpose of bifurcation analysis or development of controllers is computationally prohibitive $[49,51,54,55,57]$. Other fluid dynamics applications include shockwave-turbulent boundary layer interactions [53], cavity flows [105, 108], detonation waves [109] and jets [110]. The method has also been applied to problems where the underlying governing equations are not well-described, such as epidemiology [58], neuroscience [59], and computer vision problems such as foreground/background separation in video streams [60].

The architecture of DMD allows for methodological extensions that allow for even wider adoption by experimentalists, engineers, and scientists. Innovative measurement strategies utilizing recent developments in compressive sensing [111] allow for experimentalists to collect less data in time [57] and in space $[63,112,113]$ while still 
reconstructing relevant dynamic characteristics. Other relevant innovations include memory-efficient algorithms for BPOD based on DMD [102], error and uncertainty analysis of growth rates [114], and more accurate low-order models via optimal mode decomposition [115]. DMD has also been extended to include inputs and control expanding the method to complex systems that allow for exogenous inputs and nonautonomous dynamical systems [61]. DMD and DMDc have been connected to system identification methods like ERA $[51,61]$ and the Observer Kalman Identification method (OKID) [61]. In Sect. 3, we highlight how this equation-free architecture can be generalized to allow inputs and control.

Despite the growing popularity, a number of open challenges remain for DMDbased methods. For example, the application of DMD to data gathered from a complex system with a single traveling wave produces a set of oscillatory modes and frequencies that help characterize the wave data, but produces incorrect predictive models. Long-term predictions constructed from data from nonlinear systems is difficult for reduced-order models built with DMD modes and frequencies, due in general to the finite-dimensional, linear approximation of the Koopman operator inherent in the DMD formulation. Recent innovations that augment the data with kernel transformations, which increases the dimensionality of the input data effectively adding more measurements, may help solve these challenges and provide a far better approximation of the infinite-dimensional Koopman operator $[69,70]$. These innovations, though, introduce computational difficulties in automatically choosing the appropriate kernel. Ultimately, data-driven prediction of nonlinear systems is an open challenge to all methods. DMD and it's innovative variants may help solve a number of these important issues while also providing insight into a large-variety of modern applications.

\subsubsection{Implementation}

Consider a set of snapshots $\mathbf{x}_{j}$ where $j=1,2, \ldots, m$ which are collected from a complex system. Instead of constructing a single data matrix as in the case of POD, DMD constructs a a snapshot matrix $\mathbf{X}$ and a time-shifted snapshot matrix $\mathbf{X}^{\prime}$ :

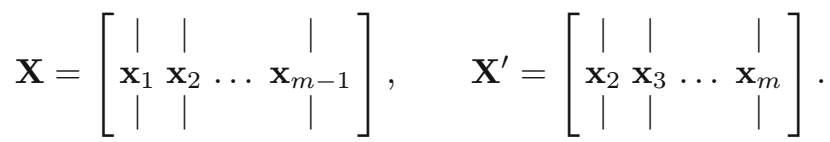

For these data matrices, define the operator

$$
\mathbf{A} \triangleq \mathbf{X}^{\prime} \mathbf{X}^{\dagger}
$$

where ${ }^{\dagger}$ is the pseudoinverse. The DMD of the measurement matrix pair $\left(\mathbf{X}, \mathbf{X}^{\prime}\right)$ is given by the eigendecomposition of $\mathbf{A}$. The dynamic modes and eigenvalues are the eigenvectors and eigenvalues of $\mathbf{A}$. The data $\mathbf{x}_{j}$ need not be collected from a single trajectory from phase space [51], but can instead be collected from a diverse set of locations as long as the $j$ th column of $\mathbf{X}$ is temporally related to the $j$ th column of $\mathbf{X}^{\prime}$.

To solve for the pseudoinverse, we compute the SVD of $\mathbf{X}$ with truncation value $r$ giving $\mathbf{X} \approx \tilde{\mathbf{U}} \tilde{\boldsymbol{\Sigma}} \tilde{\mathbf{V}}^{*}$. The operator $\mathbf{A}$ can then be approximated by

$$
\mathbf{A} \approx \mathbf{X}^{\prime} \tilde{\mathbf{V}} \tilde{\mathbf{\Sigma}}^{-1} \tilde{\mathbf{U}}^{*}
$$

For applications with a large number of states $n$, the computation of $\mathbf{A}$ can be infeasible since $\mathbf{A} \in \mathbb{R}^{n \times n}$. Instead, a low-rank approximation of $\mathbf{A}$ can be computed 
using the POD modes $\tilde{\mathbf{U}}$ of $\mathbf{X}$

$$
\tilde{\mathbf{A}}=\tilde{\mathbf{U}}^{*} \mathbf{X}^{\prime} \tilde{\mathbf{V}} \tilde{\mathbf{\Sigma}}^{-1},
$$

allowing a smaller memory footprint where $\tilde{\mathbf{X}} \in \mathbb{R}^{r \times r}$. The eigenvectors and eigenvalues of $\tilde{\mathbf{A}}$, given by the standard formulation $\tilde{\mathbf{A}} \mathbf{W}=\mathbf{W} \Lambda$, can be used to discover the dynamic modes and eigenvalues of $\mathbf{A}$ :

$$
\mathbf{\Phi}=\mathbf{X}^{\prime} \tilde{\mathbf{V}} \tilde{\boldsymbol{\Sigma}}^{-1} \mathbf{W},
$$

where $\boldsymbol{\Phi}$ are the dynamic modes and $\Lambda$ are the eigenvalues of $\mathbf{A}$ for $\lambda \neq 0$. If $\lambda=0$, then $\mathbf{\Phi}=\tilde{\mathbf{U} W}$ [51]. A solution of the system can be constructed through linear superposition of the dynamic modes and eigenvalues

$$
\mathbf{x}_{k}=\mathbf{\Phi} \boldsymbol{\Lambda}^{k} \mathbf{b}
$$

where the initial condition is computed by projecting a data snapshot on to the dynamic mode $\mathbf{x}_{k}: \mathbf{b}=\boldsymbol{\Phi}^{\dagger} \mathbf{x}_{1}$. Note that the dynamic modes and eigenvalues can be used as a diagnostic to investigate the dynamic characteristics of the data. These same modes and eigenvalues can also be used to construct a model for prediction as seen in (23).

\subsection{Koopman operator theory}

Koopman operator theory is a powerful, popular, and distinctly promising set of ideas allowing the analysis of complex, nonlinear dynamical systems. The theory illustrates how a nonlinear dynamical system can be transformed into an infinitedimensional linear system allowing solutions of the system to be constructed with traditional linear operator methods $[48,71,72,116]$. DMD, a more recent development from 2008 [47], was shown to be a computation of the Koopman mode decomposition for linear observable functions [48]. Koopman operator theory, though, stretches back to the early twentieth century with the foundational work of Bernard Koopman on measure-preserving flows $[71,116]$. These ideas have resurfaced for a number of reasons: the seminal work of Igor Mezić et al. [72,73] has demonstrated that the theory can be utilized as a spectral analysis of nonlinear dynamical systems; we now have the computational capabilities to actually perform these analyses. There are a number of comprehensive reviews on the application of Koopman analysis [73,117]. The Koopman/DMD connections have led to a number of investigations within fluid dynamics $[48,73]$ including a bifurcation analysis of flow past a cylinder [56].

The connections between DMD and Koopman have also inspired a number of engineering-oriented, methodological advances. DMD has been shown to be a method that implements a numerical framework for Koopman mode decomposition on a finitedimensional set of linear observable functions. For numerical fluids problems, this practically translates to using the entire fluid-flow state as the data for DMD, see [51] for an example. Recent advances have illustrated how augmenting the data for DMD via a larger set of observable functions including nonlinear functions offers a richer input data and can more accurately capture the nonlinearities of the data $[69,70]$. Choosing the correct set of nonlinear observable functions to augment the data set is an exciting, open research question [75]. Koopman theory is also being extended for the explicit purpose of constructing a set of input-output models for control $[75,76]$. The later sections of this review highlight research that generalizes Koopman and DMD for the characterization of input-output models of nonlinear systems. 


\subsubsection{Implementation}

We briefly describe the formulation of Koopman operator theory for the analysis of nonlinear dynamical systems. Consider the discrete nonlinear dynamical system:

$$
\mathbf{x}_{k+1}=\mathbf{f}\left(\mathbf{x}_{k}\right)
$$

evolving on a smooth manifold $\mathcal{M}$ where $\mathbf{x}_{k} \in \mathcal{M}$. The map $\mathbf{f}$ is from $\mathcal{M}$ to itself and $k$ is an integer index. There is a continuous-time formulation that could also be presented, but here we focus on the discrete perspective in line with engineering-oriented applications. We define a set of scalar valued observable functions $g: \mathcal{H} \rightarrow \mathbb{R}$, which forms an infinite-dimensional Hilbert space. We define this set as the Lebesque squareintegrable functions on $\mathcal{H}$. The Koopman operator $\mathcal{K}$ acts on this set of observable functions as:

$$
\mathcal{K} g(\mathbf{x}) \triangleq g(\mathbf{f}(\mathbf{x}))
$$

The Koopman operator $\mathcal{K}$ is linear and infinite-dimensional. Linear operator theory allows the eigendecomposition of $\mathcal{K}$ :

$$
\mathcal{K} \varphi_{j}(\mathbf{x})=\lambda_{j} \varphi_{j}(\mathbf{x}), \quad j=1,2, \ldots, \infty .
$$

The definition of the Koopman operator and the infinite expansion can be used to represent a vector-valued observable function $\mathbf{g}: \mathcal{M} \rightarrow \mathbb{R}^{n_{y}}$ in terms of the span of $\mathcal{K}$ given by the eigenfunctions $\varphi_{j}$ :

$$
\mathbf{g}(\mathbf{x})=\left[\begin{array}{c}
g_{1}(\mathbf{x}) \\
g_{2}(\mathbf{x}) \\
g_{3}(\mathbf{x}) \\
\vdots \\
g_{n_{y}}(\mathbf{x})
\end{array}\right]=\sum_{j=1}^{\infty} \varphi_{j}(\mathbf{x}) \mathbf{v}_{j}
$$

where the vector valued coefficients $\mathbf{v}_{j}$ are called Koopman modes. Applying the Koopman operator to $\mathbf{g}(\mathbf{x})$ gives

$$
\mathcal{K} \mathbf{g}(\mathbf{x})=\mathbf{g}(\mathbf{f}(\mathbf{x}))=\sum_{j=1}^{\infty} \lambda_{j} \varphi_{j}(\mathbf{x}) \mathbf{v}_{j}
$$

where the Koopman eigenvalues $\lambda_{j}$ provide the growth/decay and frequency content of the Koopman modes, $\mathbf{v}_{j}$. The Koopman modes correspond to the dynamic modes of DMD. The eigenfunctions $\varphi_{j}(\mathbf{x})$ of the Koopman operator are considered constants and are typically absorbed in to each of the dynamic modes for DMD $[48,51]$.

The Koopman operator can be viewed as a propagator on observable functions. In fact, the Koopman operator is a propagator for all observable functions in $\mathcal{H}$. To actually compute a representation of the operator $\mathcal{K}$, though, a much smaller set of observable functions are typically chosen. The observable functions for DMD are usually the identity, providing an approximate operator $\mathbf{A}$ on only the measurements available. The finite-dimensional approximations of the Koopman operator with more observable functions than identity will be denoted by $\mathbf{K}$, and is explored in Sect. 4 and 5. Recent work has illustrated the benefits of augmenting the observables with nonlinear functions $[69,70,75]$. We believe this is an exciting research direction as it presents theoreticians and practitioners a challenge in how to choose observable functions. 


\section{Dynamic mode decomposition with control}

The characterization and control of high-dimensional, complex systems remains a challenging task. DMDc is a recent methodological extension of the DMD equationfree architecture developed to analyze complex systems that also have inputs and disturbances [61]. The method utilizes measurements of the system and the applied external control inputs in order to disambiguate the underlying dynamics and the impact of inputs. DMD and DMDc are related to system identification methods such as ERA and OKID [27,37], which were originally developed for linear systems with few measurements $[51,61]$. DMDc inherits a number of the advantageous characteristics of DMD. The method operates on snapshot data and handles large numbers of measurements from complex systems. DMDc was recently applied to a rapidly pitching airfoil to predict forces and pressures [118]. Further, DMD extensions, similar to DMDc, are utilizing techniques from the subspace identification community in order to find low-order models of wind-farms [119]. Combining the control architecture with the advantages of DMD offers an equation-free control strategy in complex systems, computationally tractable for experimentalists and theoreticians. In this section, we highlight the formulation and apply the method on a high-dimensional system with inputs.

\subsection{Method: DMDc on state and input data}

Consider the dynamical system with inputs

$$
\mathbf{x}_{k+1} \approx \mathbf{A x}_{k}+\mathbf{B} \mathbf{u}_{k}
$$

where $\mathbf{x}_{j} \in \mathbb{R}^{n}, \mathbf{u}_{j} \in \mathbb{R}^{l}, \mathbf{A} \in \mathbb{R}^{n \times n}$, and $\mathbf{B} \in \mathbb{R}^{n \times l}$. Note that the relationship in (29) does not need to hold exactly. The snapshot matrices $\mathbf{X}$ and $\mathbf{X}^{\prime}$ are collected similar to DMD. The control input snapshots compose an input data matrix:

$$
\mathbf{\Upsilon}=\left[\begin{array}{cccc}
\mid & \mid & & \mid \\
\mathbf{u}_{1} & \mathbf{u}_{2} & \ldots & \mathbf{u}_{m-1} \\
\mid & \mid & & \mid
\end{array}\right]
$$

Using the snapshot matrices, (29) can be rewritten in matrix form:

$$
\begin{gathered}
\mathbf{X}^{\prime} \approx \mathbf{A X}+\mathbf{B} \boldsymbol{\Upsilon}, \\
\mathbf{X}^{\prime} \approx \mathbf{G} \boldsymbol{\Omega},
\end{gathered}
$$

where $\mathbf{G}=\left[\begin{array}{ll}\mathbf{A} & \mathbf{B}\end{array}\right]$ and $\boldsymbol{\Omega}=\left[\begin{array}{l}\mathbf{X} \\ \mathbf{\Upsilon}\end{array}\right]$. The Dynamic Mode Decomposition with control of the measurement trio $\left(\mathbf{X}, \mathbf{X}^{\prime}, \mathbf{\Upsilon}\right)$ is the eigendecomposition of the operator $\mathbf{A}$ defined by the following

$$
\mathbf{G}=[\mathbf{A} \mathbf{B}]=\mathbf{X}^{\prime} \mathbf{\Omega}^{\dagger} .
$$

We seek a best-fit solution of the operator G. The SVD is utilized to compute the pseudoinverse on the augmented data matrix giving $\boldsymbol{\Omega} \approx \tilde{\mathbf{U}} \tilde{\boldsymbol{\Sigma}} \tilde{\mathbf{V}}^{*}$. The truncation value of the SVD for $\Omega$ will be defined as $p$ which should be larger than a truncation value for $\mathbf{X}^{\prime}$. The approximation is

$$
\mathbf{G} \approx \overline{\mathbf{G}}=\mathbf{X}^{\prime} \tilde{\mathbf{V}} \tilde{\mathbf{\Sigma}}^{-1} \tilde{\mathbf{U}}^{*}
$$


where $\mathbf{G} \in \mathbb{R}^{n \times(n+l)}$. A and $\mathbf{B}$ can be found by breaking the unitary operator $\tilde{\mathbf{U}}$ in to two separate components:

$$
\begin{aligned}
& {\left[\begin{array}{ll}
\mathbf{A}, & \mathbf{B}
\end{array}\right] \approx\left[\begin{array}{ll}
\overline{\mathbf{A}}, & \overline{\mathbf{B}}
\end{array}\right]} \\
& \approx\left[\mathbf{X}^{\prime} \tilde{\mathbf{V}} \tilde{\boldsymbol{\Sigma}}^{-1} \tilde{\mathbf{U}}_{1}^{*}, \quad \mathbf{X}^{\prime} \tilde{\mathbf{V}} \tilde{\boldsymbol{\Sigma}}^{-1} \tilde{\mathbf{U}}_{2}^{*}\right]
\end{aligned}
$$

where $\tilde{\mathbf{U}}_{1} \in \mathbb{R}^{n \times p}, \tilde{\mathbf{U}}_{2} \in \mathbb{R}^{l \times p}$, and $\tilde{\mathbf{U}}^{*}=\left[\tilde{\mathbf{U}}_{1}^{*} \tilde{\mathbf{U}}_{2}^{*}\right]$. The nonsquare operator $\mathbf{G}$ is size $n \times n+l$ which could be computationally prohibitive to compute. A reduced order version can be computed, but not with the left singular vectors of $\boldsymbol{\Omega}$. To find a linear transformation for a reduced order subspace on which $\mathbf{x}$ evolves, we utilize a subspace constructed from the output data matrix $\mathbf{X}^{\prime}$. The SVD of $\mathbf{X}^{\prime}$ is given by $\hat{\mathbf{U}} \hat{\boldsymbol{\Sigma}} \hat{\mathbf{V}}^{*}$ where the truncation value is $r$ and $\hat{\mathbf{U}} \in \mathbb{R}^{n \times r}, \hat{\boldsymbol{\Sigma}} \in \mathbb{R}^{r \times r}$, and $\hat{\mathbf{V}}^{*} \in \mathbb{R}^{r \times m-1}$. The following reduced-order approximations of $\mathbf{A}$ and $\mathbf{B}$ can be computed:

$$
\begin{gathered}
\tilde{\mathbf{A}}=\hat{\mathbf{U}}^{*} \overline{\mathbf{A}} \hat{\mathbf{U}}=\hat{\mathbf{U}}^{*} \mathbf{X}^{\prime} \tilde{\mathbf{V}} \tilde{\boldsymbol{\Sigma}}^{-1} \tilde{\mathbf{U}}_{1}^{*} \hat{\mathbf{U}} \\
\tilde{\mathbf{B}}=\hat{\mathbf{U}}^{*} \overline{\mathbf{B}}=\hat{\mathbf{U}}^{*} \mathbf{X}^{\prime} \tilde{\mathbf{V}} \tilde{\mathbf{\Sigma}}^{-1} \tilde{\mathbf{U}}_{2}^{*}
\end{gathered}
$$

where $\tilde{\mathbf{A}} \in \mathbb{R}^{r \times r}$ and $\tilde{\mathbf{B}} \in \mathbb{R}^{r \times l}$. The relationship between the eigenvectors of $\tilde{\mathbf{A}}$ and the dynamic modes of $\mathbf{A}$ is:

$$
\phi=\mathbf{X}^{\prime} \tilde{\mathbf{V}} \tilde{\boldsymbol{\Sigma}}^{-1} \tilde{\mathbf{U}}_{1}^{*} \hat{\mathbf{U}} \mathbf{w},
$$

which is slightly modified from the dynamic modes of DMD.

\subsection{Example: Disambiguating dynamics from inputs}

In this example, we construct a high-dimensional non-autonomous dynamical systems with a low-rank solution. A similar example was also described in $[61,63]$. The spatial domain is of size $128 \times 128$ with periodic boundaries. Five two-dimensional fourier modes are chosen at random to be nonzero. The dynamical system on these five fourier modes is also chosen at random with the constraint that the eigenvalues for each mode are near the unit circle with a temporally evolving frequency and a small damping rate. Five oscillating spatial fourier modes creates a high-dimensional dynamical system in space. A random disturbance is applied as an external input to a single spatial pixel. A snapshot of one realization of this dynamical system is illustrated on the torus is shown in Fig. 2.

After the dynamical system is constructed and data collected, we use DMDc to disambiguate the underlying dynamics with the external inputs. The data matrices $\left(\mathbf{X}, \mathbf{X}^{\prime}, \mathbf{\Upsilon}\right)$ are of size $128^{2} \times 200$. DMDc discovers the underlying temporal frequencies and damping coefficients of the dynamical systems. The middle panel of Fig. 2 shows that DMD would incorrectly predict eigenvalues outside of the unit circle, indicating growth of the dynamical system. DMDc accurately finds the eigenvalues of the underlying dynamical system. Moreover, the dynamic modes, shown in right panel, are accurately reconstructed by DMDc. 

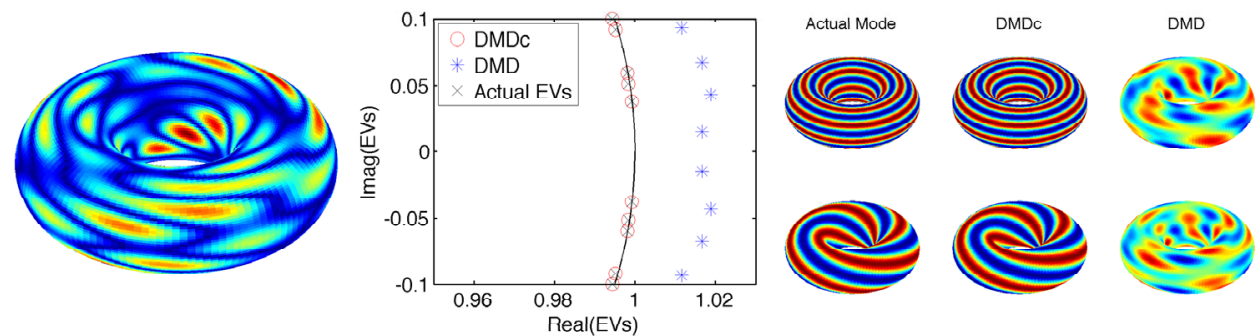

Fig. 2. Numerical results using DMD and DMDc on spatial-temporal data given in the left plot. The middle plot shows the actual eigenvalues with the computed eigenvalues from DMD and DMDc. The right plot shows the actual dynamic modes, the DMD modes, and the DMDc modes plotted on the torus.

\section{Koopman observable subspaces and finite linear representations of nonlinear dynamical systems for control}

Koopman operator theory has primarily focused on the characterization and identification of important dynamical properties of complex systems [48,51,72]. There is also significant interest in discovering a finite-dimensional approximation of the Koopman operator that acts as a propagator for the observable functions. This approximate operator could be used to predict the future observables allowing for the design of controllers on the set of observables [75]. However, many dynamical systems do not admit a finite-dimensional Koopman-invariant subspace that includes direct measurements of the original state. If the nonlinear system contains more than a single fixed point, a finite-dimensional Koopman-invariant subspace explicitly containing the original state does not exist [75]. In this section, we highlight how to obtain a Koopman-invariant subspace for a nonlinear system.

\subsection{Method: Koopman observable subspaces containing the state}

A Hilbert space $\mathcal{H}$ can be spanned by an infinite set of basis functions $y_{1}(\mathbf{x}), y_{2}(\mathbf{x}), \ldots$, and any finite subset of these observable functions will form a finite-dimensional subspace. A Koopman-invariant observable subspace is defined as the span of a subset of observable functions $y_{s_{1}}, y_{s_{2}}, \ldots, y_{s_{m}}$ such that any function $g(\mathbf{x})$ in this subspace

$$
g=\alpha_{1} y_{s_{1}}+\alpha_{2} y_{s_{2}}+\cdots+\alpha_{m} y_{s_{m}}
$$

remains in this subspace after being acted on by the the Koopman operator:

$$
\mathcal{K} g=\beta_{1} y_{s_{1}}+\beta_{2} y_{s_{2}}+\cdots+\beta_{m} y_{s_{m}} .
$$

The operator $\mathcal{K}$ can be restricted to this subspace of observable functions, in which case it defines a finite-dimensional operator $\mathbf{K}$ and linear dynamical system, even though the observables are nonlinear. Any subset of eigenfunctions of the Koopman operator form an invariant subspace, by definition. However, these eigenfunctions may be arbitrarily complex, as they are intricately related to the phase space geometry of the dynamical system [120-122].

Similar to DMD, we would like the Koopman-invariant subspaces to include the state variables $\mathbf{x}$ directly as observables. Then the Koopman operator restricted to this subspace provides a linear propagator for the original state. Including the state 
variables requires the right hand side of the nonlinear vector field to be included in the subspace:

$$
\frac{d}{d t}\left[\begin{array}{c}
x_{1} \\
x_{2} \\
\vdots \\
x_{n}
\end{array}\right]=\left[\begin{array}{c}
f_{1}\left(x_{1}, x_{2}, \cdots, x_{n}\right) \\
f_{2}\left(x_{1}, x_{2}, \cdots, x_{n}\right) \\
\vdots \\
f_{n}\left(x_{1}, x_{2}, \cdots, x_{n}\right)
\end{array}\right] .
$$

We may define the first $n$ observable functions to be the components of the state variable $\mathbf{x}$, and the remaining observables are chosen to span the right hand side of (39):

$$
f_{k}\left(x_{1}, x_{2}, \cdots, x_{n}\right)=c_{k, 1} y_{s_{1}}+c_{k, 2} y_{s_{2}}+\cdots+c_{k, m} y_{s_{m}}, .
$$

However, it is unclear whether or not the time derivative of these additional nonlinear observable functions can be expressed in terms of the functions in the subspace, or whether they will require additional observable functions to be included in the span:

$$
\frac{d}{d t}\left[\begin{array}{c}
y_{1} \\
y_{2} \\
\vdots \\
y_{n} \\
y_{n+1} \\
\vdots \\
y_{m}
\end{array}\right]=\left[\begin{array}{ccccccc}
c_{1,1} & c_{1,2} & \cdots & c_{1, n} & c_{1, n+1} & \cdots & c_{1, m} \\
c_{2,1} & c_{2,2} & \cdots & c_{2, n} & c_{2, n+1} & \cdots & c_{2, m} \\
\vdots & \vdots & \ddots & \vdots & \vdots & \ddots & \vdots \\
c_{n, 1} & c_{n, 2} & \cdots & c_{n, n} & c_{n, n+1} & \cdots & c_{n, m} \\
? & ? & \cdots & ? & ? & \cdots & ? \\
\vdots & \vdots & \ddots & \vdots & \vdots & \ddots & \vdots \\
? & ? & \cdots & ? & ? & \cdots & ?
\end{array}\right]\left[\begin{array}{c}
y_{1} \\
y_{2} \\
\vdots \\
y_{n} \\
y_{n+1} \\
\vdots \\
y_{m}
\end{array}\right]
$$

In general, the first $n$ rows are a direct representation of the vector field from (39). The next $m-n$ rows can be computed analytically by successively computing $\frac{d}{d t} y_{k}$ for $k>m$. If the number of observable functions continues to grow recursively, the dynamical system does not close to a finite-dimensional representation on a Koopman invariant subspace. See [75] for more details about the challenge of closure for these dynamical systems.

\subsection{Example: A Koopman perspective on a nonlinear dynamical system}

In this example, we illustrate the method on a dynamical system with a slow manifold illustrated in Fig. 3. The system is given by:

$$
\begin{gathered}
\dot{x}_{1}=\mu x_{1} \\
\dot{x}_{2},=\lambda\left(x_{2}-x_{1}^{2}\right) .
\end{gathered}
$$

Choosing the observable functions $\mathbf{y}=\left[\begin{array}{lll}y_{1} & y_{2} & y_{3}\end{array}\right]^{T}=\left[\begin{array}{lll}x_{1} & x_{2} & x_{1}^{2}\end{array}\right]^{T}$ results in a Koopman-invariant subspace that explicitly includes the state. The induced linear system obtained by restricting the Koopman operator to this subspace is given by:

$$
\frac{d}{d t}\left[\begin{array}{l}
y_{1} \\
y_{2} \\
y_{3}
\end{array}\right]=\left[\begin{array}{ccc}
\mu & 0 & 0 \\
0 & \lambda & -\lambda \\
0 & 0 & 2 \mu
\end{array}\right]\left[\begin{array}{l}
y_{1} \\
y_{2} \\
y_{3}
\end{array}\right] .
$$

Consider the embedding of the nonlinear dynamical system into a higher-dimensional, linear observable subspace. The right panel of Fig. 3 illustrates how the quadratic 

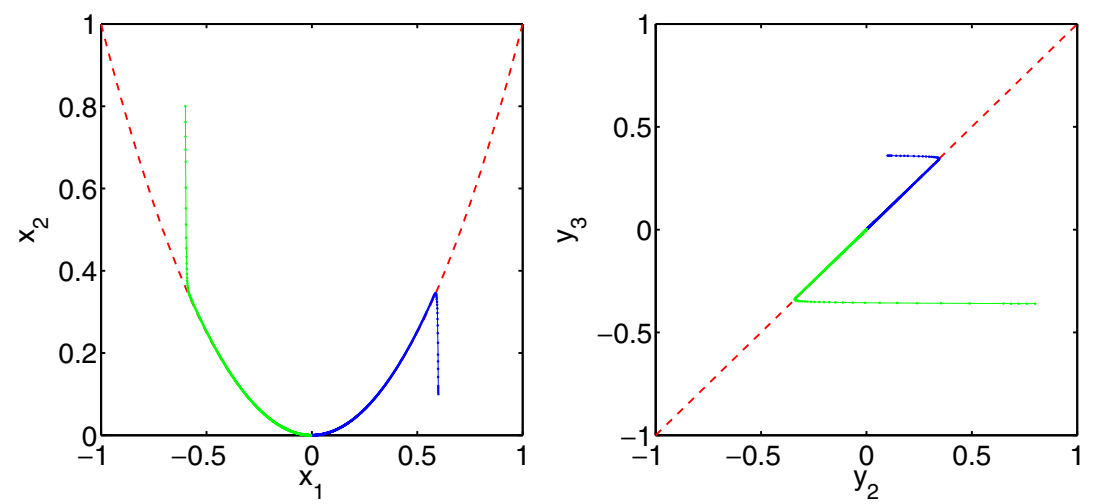

Fig. 3. The left panel shows the slow manifold (in dashed red line) with two trajectories of (43). In the right panel, the Koopman-invariant observable subspace is illustrated. The dashed red line is the slow manifold between observables $y_{2}$ and $y_{3}$. The blue and green lines are trajectories with same initial conditions as the left panel.

nonlinearity in the system can be linearized (dashed red line). Two trajectories are represented for the underlying nonlinear system in the left panel. The same two trajectories are shown in the linearized system in the right panel. The restricted Koopman operator of (43) can be used to construct a controller on the observables for some types of inputs [75]. The dynamical system investigated in this section has a single fixed point. When more than one fixed point exists, the finite-dimensional closure is not possible for subspaces containing the state, creating the need for an ever-growing set of rows in (41) to represent the new nonlinearities.

For this example, the nonlinear embedding is known a-priori allowing a clear depiction of a Koopman-invariant subspace for a nonlinear dynamical system. In general, the nonlinear embedding is not known, requiring an exploration of nonlinear observables. Further, incorrect observable functions can create a misleading characterization of the dynamics and incorrect predictive models. One current line of research is focused on augmenting the input data with nonlinear observable functions based on kernel expansions, thus spanning a larger space of nonlinear functions [69]. Alternatively, another research perspective is focused on identifying a sparse number of nonlinear functions from a large library of possible functions to fit the nonlinear dynamics [123]. Choosing the correct set of observable functions is a fundamental and open-challenge for using Koopman operator theory for control.

\section{Koopman with inputs and control}

Koopman with inputs and control (KIC) is a generalization of Koopman operator theory allowing for the analysis of complex systems with inputs and control [76]. DMDc restricts the observable functions to the identity map on the state. The extension of Koopman operator theory allows for the consideration of a wider set of observable functions, which can include nonlinear observable functions of the state, the input, and mixed terms. Similar to the close connections of DMDc to subspace identification methods, the algorithm for KIC has strong numerical similarities with system identification methods such as nonlinear autoregressive exogenous model (NARX) [124]. This generalization of Koopman operator theory, though, is a step toward data-driven characterization and control of complex input-output systems from an operator theoretic perspective. We describe the formulation of KIC and 
illustrate how the infinite-dimensional operator can be approximated for a nonlinear, finite-dimensional dynamical system with inputs.

\subsection{Method: Koopman with inputs and control}

Consider the following non-autonomous dynamical system

$$
\mathbf{x}_{k+1}=\mathbf{f}\left(\mathbf{x}_{k}, \mathbf{u}_{k}\right)
$$

where $\mathbf{x} \in \mathcal{M}$ and $\mathbf{u} \in \mathcal{N}$ where both $\mathcal{M}$ and $\mathcal{N}$ are smooth manifolds. The scalar-valued observable functions are now dependent on the state and the inputs $g: \mathcal{M} \otimes \mathcal{N} \rightarrow \mathbb{R}$. The set of observable functions comprise a Hilbert space $\mathcal{H}$. Similar to the previous section on Koopman-invariant-subspaces, $\mathcal{H}$ can be partitioned in to different subspaces. For example, three separate Hilbert subspaces can be constructed where the functions $g(\mathbf{x}, \mathbf{u})=g(\mathbf{x})$ are in $\mathcal{H}_{X}, g(\mathbf{x}, \mathbf{u})=g(\mathbf{u})$ are in $\mathcal{H}_{U}$, and finally the complement $\mathcal{H}_{X U}$ which contain observable functions that offer mixed terms such as $g_{\mathbf{x}, \mathbf{u}}=x_{1} u_{1}$. The partitioning is sufficiently general that is also includes linear identity observables, i.e. $g(\mathbf{x})=x_{1}$ where $x_{1}$ is the first element of $\mathbf{x}$, in addition to nonlinear observables i.e. $g(\mathbf{x})=x_{1}^{2}$. Considering only the subspace of linear observable functions leads to the formulation of DMDc [76].

The Koopman operator with inputs and control $\mathcal{K}: \mathcal{H} \rightarrow \mathcal{H}$ acts on the Hilbert space of observable functions given by the following:

$$
\mathcal{K} g(\mathbf{x}, \mathbf{u}) \triangleq g(\mathbf{f}(\mathbf{x}, \mathbf{u}), *)
$$

where $*$ indicates a choice of definition. If $*=\mathbf{u}$, then the definition allows for the recovery of dynamics on the inputs, as is the case for state feedback controllers. If $*=0$, then the operator does not specify dynamics on the inputs as in the case with exogenous forcing and random disturbances. Restricting $\mathcal{H}$ to linear observables on the state and inputs and choosing $*=0$ reduces KIC to DMDc. The linear characteristics of the Koopman operator allow us to perform an eigendecomposition of $\mathcal{K}$ given in the standard form:

$$
\mathcal{K} \varphi_{j}(\mathbf{x}, \mathbf{u})=\lambda_{j} \varphi_{j}(\mathbf{x}, \mathbf{u}), \quad j=1,2, \ldots
$$

The operator is now spanned by eigenfunctions that are defined by the inputs and state. Using the infinite expansion shown in Eq. (26), the observable functions $g_{j}$ can be rewritten in terms of the right eigenfunctions $\varphi_{j}$,

$$
\mathbf{g}(\mathbf{x}, \mathbf{u})=\left[\begin{array}{c}
g_{1}(\mathbf{x}, \mathbf{u}) \\
g_{2}(\mathbf{x}, \mathbf{u}) \\
\vdots \\
g_{n_{y}}(\mathbf{x}, \mathbf{u})
\end{array}\right]=\sum_{j=1}^{\infty} \varphi_{j}(\mathbf{x}, \mathbf{u}) \mathbf{v}_{j}
$$

where $n_{y}$ is the number of measurements. The new Koopman operator can be applied to this representation of the measurement

$$
\mathcal{K} \mathbf{g}(\mathbf{x}, \mathbf{u})=\mathbf{g}(\mathbf{f}(\mathbf{x}, \mathbf{u}), \mathbf{u})=\sum_{j=1}^{\infty} \lambda_{j} \varphi_{j}(\mathbf{x}, \mathbf{u}) \mathbf{v}_{j}
$$

A number of promising research directions result from this definition of KIC. If $*=0$ in (45), a Koopman operator can be constructed on a restricted set of observable 
functions that include nonlinear functions of the state, inputs, and mixed terms. The output of the Koopman operator is only the state. For a DMD-like computation on real data, the Koopman operator becomes a nonsquare operator allowing for a large set of observable nonlinear functions without trying to fit input dynamics. This perspective can be taken one step further to propose different domains and ranges for a restricted Koopman operator. This introduces a number of new and exciting challenges such as how to pick the right observable functions for the states and inputs for a specific application. Recent work has utilized sparsity-promoting methods to pick the right nonlinear functions for characterization of the dynamical systems [125].

\subsection{Example: Recovering nonlinear dynamical systems from data}

In this example, we illustrate how inputs can be included to discover an approximation of the Koopman operator that is a propagator of the system observables even when exogenous forcing is present. Here, we have chosen an underlying system that has a Koopman-invariant subspace. Similar to the notation for Koopman operator theory without inputs, we denote the finite approximation with inputs by $\mathbf{K}$. An input term $\alpha u$ is included that consists of random disturbances. We choose the definition in (45) to be $*=0$. Consider the system

$$
\left[\begin{array}{c}
y_{1} \\
y_{2} \\
y_{3} \\
0
\end{array}\right]_{k+1}=\left[\begin{array}{cccc}
\mu & 0 & 0 & 0 \\
0 & \lambda & -\lambda & \alpha \\
0 & 0 & 2 \mu & 0 \\
0 & 0 & 0 & 0
\end{array}\right]\left[\begin{array}{l}
y_{1} \\
y_{2} \\
y_{3} \\
\gamma_{1}
\end{array}\right]_{k} \text { for }\left[\begin{array}{l}
y_{1} \\
y_{2} \\
y_{3} \\
\gamma_{1}
\end{array}\right]=\left[\begin{array}{c}
x_{1} \\
x_{2} \\
x_{1}^{2} \\
u
\end{array}\right]
$$

where $\lambda=.5, \mu=2$, and $\alpha=2$. The initial condition is $\mathbf{x}=[5,2]^{T}$. To show how to discover this Koopman operator from data, we collect a ten snapshots of data from the system given an input data matrix $\boldsymbol{\Omega}$ and output data $\mathbf{X}^{\prime}$. The first few snapshots of each data matrix gives the following:

$$
\boldsymbol{\Omega}=\left[\begin{array}{cccc}
5 & 10 & 20 & 40 \\
2 & -11.3 & 54.7 & -226.4 \\
25 & 100 & 400 & 1600 \\
.1 & .5 & .5 & 2.2
\end{array}\right] \quad \mathbf{X}^{\prime}=\left[\begin{array}{cccc}
10 & 20 & 40 & 80 \\
-11.3 & 54.7 & -226.4 & -908.9 \\
100 & 400 & 1600 & 6400
\end{array}\right]
$$

Using the DMDc numerical procedure, the restricted Koopman operator can be exactly recovered with $\mathbf{K}=\mathbf{X}^{\prime} \mathbf{\Omega}^{\dagger}$. The tractable computational procedure discovers a linear input-output system for a nonlinear dynamical system:

$$
\begin{gathered}
\mathbf{K}=\left[\mathbf{K}_{A} \mathbf{K}_{B}\right], \\
\mathbf{y}_{k+1}=\mathbf{K}_{A} \mathbf{y}_{k}+\mathbf{K}_{B} \gamma_{k} .
\end{gathered}
$$

The example can be changed to investigate both other definitions of KIC, including $*=\mathbf{u}_{k+1}$, and systems with a higher-dimensional state, as in Sect. 3.2. For example, the inputs would have dynamics based on feedback controllers or possibly multi-scale modeling.

Similar to the example in Sect. 4, the nonlinear embedding is known in (49). We specifically choose an analytic example to illustrate KIC on a nonlinear dynamical system. In general for equation-free modeling, the nonlinear observables will not be conveniently available. Current research is focused on how to discover the nonlinear functions from a large library [123] or by transforming the problem using kernel functions [69]. 


\section{Discussion and conclusions}

The characterization and control of high-dimensional, complex systems is becoming increasingly important as we engineer and build ever larger systems such as the electrical grid, internet infrastructure, distribution of goods, and coordinated autonomous driving platforms. Many of these systems do not have well-characterized, physicsbased governing equations that lend themselves to traditional analyses. Our ability to collect high-fidelity data and perform targeted experiments with these systems, though, offers significant opportunities for data-driven, equation-free modeling to discover low-dimensional models of the dynamics. We highlight that recent adaptations of these data-driven methods for handling systems with inputs offers a promising future for the design of objective-based controllers for these equation-free models.

We have offered two perspectives on analyzing complex systems: model-based dimensionality reduction and equation-free methods. The model-based dimensionality reduction has a substantial and rich history with POD and Galerkin projections $[2,6]$. Balanced POD, a recent innovation, inherits advantages from POD on high-dimensional data while balancing important input-output characteristics such as controllability and observability $[29,34-36]$. BPOD requires both the direct and adjoint calculations. Equation-free methods such as DMD and Koopman operator theory operate solely on measurement data, are connected to the analysis of nonlinear systems, and scale favorably computationally to a large number of measurements. These equation-free methods have only recently been extended to handle input-output systems $[61,75,76,118]$.

We began the article with a review of model-based dimensionality reduction and equation-free methods including the context and formulation. In dimensionality reduction, POD and Galerkin methods are presented, followed by the BPOD innovations around handling high-dimensional input-output systems. We then consider the equation-free methods of DMD and Koopman operator theory for autonomous dynamical systems.

To demonstrate how data-driven, equation-free methods can be extended to handle input-output systems, three examples are presented that generalize DMD and Koopman operator theory. The first example illustrates how DMD can be extended to handle input data. The inclusion of input data allows DMDc to disambiguate the effects of the underlying dynamics and the exogenous forcing. The second two examples involve methodologically extending Koopman operator theory to handle nonlinear data and inputs. Establishing that systems with a single fixed-point can often be described by a restricted Koopman invariant subspace that includes linear measurements allows for a nonlinear vector-field to be transformed in to a finite-dimensional linear model that propagates the original state. Linearizing the model allows for standard control-theoretic techniques to construct objective-based controllers. The final example illustrates how to include inputs within the Koopman architecture. A Koopman operator can be discovered that disambiguates the effect of nonlinear dynamics from inputs.

JLP would like to thank Bill and Melinda Gates for their active support of the Institute for Disease Modeling and their sponsorship through the Global Good Fund. SLB and JNK acknowledge generous support from the Defense Advanced Research Projects Agency (DARPA contract HR0011-16-C-0016). JNK also acknowledges support from the U.S. Air Force Office of Scientific Research (FA9550-15-1-0385).

\section{References}

1. P. Holmes, J. Guckenheimer, Nonlinear Oscillations, Dynamical Systems, and Bifurcations of Vector Fields, Vol. 42 of Applied Mathematical Sciences (SpringerVerlag, Berlin, Heidelberg, 1983) 
2. J. L. Lumley, Stochastic Tools in Turbulence (Academic Press, 1970)

3. G. Berkooz, P. Holmes, J.L. Lumley, Ann. Rev. Fluid Mech. 23, 539 (1993)

4. T. Colonius, J. Freund, AIAA Paper 2002-0072 (2002)

5. T. Smith, Ph.D. thesis, Princeton University, 2003

6. P.J. Holmes, J.L. Lumley, G. Berkooz, C.W. Rowley, Turbulence, coherent structures, dynamical systems and symmetry, 2nd edn. Cambridge Monographs in Mechanics (Cambridge University Press, Cambridge, England, 2012)

7. M. Rathinam, L. Petzold, SIAM J. Numer. Anal. 5, 1893 (2003)

8. B. Epureanu, L. Tang, M. Paidoussis, Int. J. Non-Linear Mech. 36, 977 (2004)

9. L.S.C. Raptopoulos, M.S. Dutra, F.A. de Noronha Castro Pinto, A.C. de Pina Filho, J. Biomech. 39, 2898 (2006)

10. D. Riskin, et al., J. Theoretical Biol. 254, 604 (2008)

11. M. Azeez, A. Vakakis, J. Sound Vib. 240, 859 (2001)

12. J. Cusuman, B. Bai, Chaos Solitons Fractals 3, 515 (1993)

13. J. Cusuman, B. Bai, Phil. Trans. Roy. Soc. of Lond. 347, 421 (1994)

14. U. Feldmann, E. Kreuzer, F. Pinto, Nonlinear Dynamics 22, 183 (2000)

15. D. Boe, J. Golinval, Structural Health Monitoring 2, 137 (2003)

16. C.W. Rowley, T. Colonius, R.M. Murray, Physica D 189, 115 (2004)

17. E. Shlizerman, E. Ding, M. Williams, J.N. Kutz, Int. J. Opt. 2012, 831604 (2012)

18. M. Williams, E. Shlizerman, J.N. Kutz, J. Opt. Soc. Am. B 27, 2471 (2010)

19. E. Ding, E. Shlizerman, J.N. Kutz, Phys. Rev. A 82, 023823 (2010)

20. B.R. Noack, K. Afanasiev, M. Morzynski, G. Tadmor, F. Thiele, J. Fluid Mech. 497, $335(2003)$

21. B. Noack, M. Morzyński, G. Tadmor, Reduced-Order Modeling for Flow Control (Springer, Vienna, 2011)

22. S. Brunton, B. Noack, ASME. Appl. Mech. Rev. 67 (2015)

23. M. Barrault, Y. Maday, N. Nguyen, A. Patera, Comptes Rendus Mathematique 9, 667 (2004)

24. S. Chaturantabut, D. Sorensen, Decision and Control Conference 2009 (2009) p. 4316

25. S. Chaturantabut, D. Sorensen, SIAM J. Numer. Anal. 50, 46 (2012)

26. B.C. Moore, IEEE Trans. Automatic Control AC-26, 17 (1981)

27. J.N. Juang, R.S. Pappa, J. Guidance Control Dyn. 8, 620 (1985)

28. S. Lall, J. Marsden, S. Glavaški, Proc. of the IFAC World Congress F (1999), p. 473

29. C.W. Rowley, Int. J. Bifurc. Chaos 15, 997 (2005)

30. P. Rapisarda, T.H.L., Automatica 47, 1721 (2011)

31. R. Winck, W. Book, Automatica 49, 3084 (2013)

32. J. Guzmán I, D. Sipp, P.J. Schmid, J. Fluid Mech. 758, 728 (2014)

33. M. Fardad, F. Lin, M. Jovanović, IEEE Trans. Automatic Control 29, 2281 (2014)

34. S. Lall, J. Marsden, S. Glavaški, Int. J. Robust Nonlinear Control 12, 519 (2002)

35. K. Willcox, J. Peraire, AIAA J. 40, 2323 (2002)

36. M. Ilak, C.W. Rowley, Phys. Fluids 20, 034103 (2008)

37. J.N. Juang, M. Phan, L.G. Horta, R.W. Longman, Identification of observer/Kalman filter Markov parameters: Theory and experiments, Technical Memorandum 104069, NASA (1991)

38. M. Phan, J.N. Juang, R.W. Longman, The J. Astronautical Sci. 40, 261 (1992)

39. M. Phan, L.G. Horta, J.N. Juang, R.W. Longman, J. Optimization Theory Appl. 79, 59 (1993)

40. J.N. Juang, Applied System Identification (Prentice Hall PTR, Upper Saddle River, New Jersey, 1994)

41. M. Forgione, X. Bombois, P. Van den Hof, Automatica 52, 118 (2015)

42. Z. Ma, S. Ahuja, C.W. Rowley, Theor. Comput. Fluid Dyn. 25, 233 (2011)

43. P. Van Overschee, B. De Moor, Automatica 30, 75 (1994)

44. S.J. Qin, Comput. Chem. Eng. 30, 1502 (2006)

45. M. Viberg, Automatica. 31, 1835 (1995) 
46. T. Katayama, Subspace Methods for System Identification (Springer-Verlag, London, 2005)

47. P.J. Schmid, J. Sesterhenn, 61st Annual Meeting of the APS Division of Fluid Dynamics (2008)

48. C.W. Rowley, I. Mezić, S. Bagheri, P. Schlatter, D.S. Henningson, J. Fluid Mech. 641, $115(2009)$

49. P.J. Schmid, J. Fluid Mech. 656, 5 (2010)

50. K.K. Chen, J.H. Tu, C.W. Rowley, J. Nonlinear Sci. 22, 887 (2012)

51. J.H. Tu, C.W. Rowley, D.M. Luchtenburg, S.L. Brunton, J.N. Kutz, J. Comput. Dyn. 1, 391 (2014)

52. G. Tissot, L. Cordier, N. Benard, B.R. Noack, Comptes Rendus Mécanique (2014)

53. M. Grilli, P. Schmid, S. Hickel, N. Adams, J. Fluid Mech. 700, 16 (2012)

54. P.J. Schmid, Experiments in Fluids 50, 1123 (2011)

55. P.J. Schmid, D. Violato, F. Scarano, Experiments in Fluids 52, 1567 (2012)

56. S. Bagheri, J. Fluid Mech. 726, 596 (2013)

57. J.H. Tu, C.W. Rowley, J.N. Kutz, J.K. Shang, Experiments in Fluids 55, 1 (2014)

58. J. Proctor, P.A. Eckhoff, Int. Health 2, 139 (2015)

59. B.W. Brunton, L.A. Johnson, J.G. Ojemann, J.N. Kutz, J. Neurosci. Methods 258, 1 (2016)

60. J. Gosek, J.N. Kutz, submitted for publication (2013)

61. J.L. Proctor, S.L. Brunton, J.N. Kutz, SIAM J Appl. Dyn. Syst. 15, 142 (2016)

62. M.R. Jovanović, P.J. Schmid, J.W. Nichols, Phys. Fluids 26, 024103 (2014)

63. S.L. Brunton, J.L. Proctor, J.H. Tu, J.N. Kutz, J. Computat. Dyn. 2, 165 (2015)

64. F. Gueniat, L. Mathelin, L. Pastur, Phys. Fluids 27, 025113 (2015)

65. J.N. Kutz, X. Fu, S.L. Brunton, [arXiv:1506.00564] (2016)

66. S.T.M. Dawson, M.S. Hemati, M.O. Williams, C.W. Rowley, Characterizing and correcting for the effect of sensor noise in the dynamic mode decomposition [arXiv: 1507.02264] (2015)

67. M.S. Hemati, C.W. Rowley, [arXiv:1502.03854] (2015)

68. M.O. Williams, C.W. Rowley, I. Mezić, I.G. Kevrekidis, Europhys. Lett. 109, 40007 (2015)

69. M.O. Williams, C.W. Rowley, I.G. Kevrekidis, [arXiv:1411.2260] (2014)

70. M.O. Williams, I.G. Kevrekidis, C.W. Rowley, J. Nonlinear Sci. 25, 1307 (2015)

71. B.O. Koopman, Proc. Natl. Acad. Sci. 17, 315 (1931)

72. I. Mezić, J. Nonlin. Dyn. 41, 309 (2005)

73. M. Budišić, R. Mohr, I. Mezić, Chaos: An Interdisciplinary J. Nonlinear Sci. 22, 047510 (2012)

74. I. Mezić, Ann. Rev. Fluid Mech. 45, 357 (2013)

75. S.L. Brunton, B.W. Brunton, J.L. Proctor, J.N. Kutz, PLoS ONE 11, 1 (2016)

76. J. Proctor, S.L. Brunton, J. Kutz [arXiv:1510.03007] (2016)

77. C. Eckart, G. Young, Psychometrika 1, 211 (1936)

78. L. Mirsky, Quart. J. Math. Oxford 211, 50 (1960)

79. G.H. Golub, C. Reinsch, Numerical Math. 14, 403 (1970)

80. A. Antoulas, D. Sorensen, Int. J. Appl. Math. Comput. Sci. 11, 1093 (2001)

81. D. Jackson, Ecology 8, 2204 (1993)

82. D. Donoho, et al., Ann. Stat. 42, 2413 (2014)

83. M. Gavish, D.L. Donoho, IEEE Trans. Inf. Theory 60, 5040 (2014)

84. K. Pearson, Philos. Mag. 2, 559 (1901)

85. H. Hotelling, J. Educ. Psychol. 24, 417 (1933)

86. E.N. Lorenz, Report 1: Statistical Forecasting Project, MIT (1956)

87. G.R. North, Report 1: Statistical Forecasting Project, MIT (1956)

88. M. Loev́e, Probability Theory (Van Nostrand, New York, 1955)

89. S.S. Ravindran, Int. J. Numerical Methods in Fluids 34, 425 (2000)

90. X. Ma, G.E. Karniadakis, J. Fluid Mech. 458, 181 (2002) 
91. B.R. Noack, K. Afanasiev, M. Morzynski, G. Tadmor, F. Thiele, J. Fluid Mech. 497, $335(2003)$

92. S. Sirisup, G.E. Karniadakis, D. Xiu, I.G. Kevrekidis, J. Comput. Phys. 207, 568 (2005)

93. G. Tadmor, O. Lehmann, B.R. Noack, M. Morzynski, Phys. Fluids 22, 034102 (2010)

94. L. Sirovich, M. Kirby, J. Opt. Soc. Am. A 4, 519 (1987)

95. V. Adamjan, D. Arov, M. Krein, Math. USSR Sbornik 15, 31 (1971)

96. K. Glover, Int. J. Contr. 39, 1115 (1984)

97. V. Adamjan, D. Arov, M. Krein, Amer. Math. Soc. Trans. 111, 133 (1978)

98. S. Bagheri, L. Brandt, D.S. Henningson, J. Fluid Mech. 620, 263 (2009)

99. S.J. Illingworth, A.S. Morgans, C.W. Rowley, J. Sound Vib. 330, 1567 (2010)

100. S. Ahuja, C.W. Rowley, J. Fluid Mech. 645, 447 (2010)

101. A.C. Or, J.L. Speyer, J. Fluid Mech. 662, 36 (2010)

102. J.H. Tu, C.W. Rowley, J. Comput. Phys. 231, 5317 (2012)

103. S.L. Brunton, B.R. Noack, Appl. Mech. Rev. 67, 050801 (2015)

104. J.R. Annoni, P.J. Seiler, in 2015 American Control Conference (IEEE, 2015), pp. $1721-1727$

105. P.J. Schmid, K.E. Meyer, O. Pust, Dynamic mode decomposition and proper orthogonal decomposition of flow in a lid-driven cylindrical cavity, PIV09-0186, 8th International Symposium on Particle Image Velocimetry (2009)

106. C.W. Gear, I.G. Kevrekidis, C. Theodoropoulos, Comput. Chem. Eng. 26, 941 (2002)

107. I.G. Kevrekidis, et al., Commun. Math. Sci. 1, 715 (2003)

108. A. Seena, H. Sung, Int. J. Heat Fluid Flow 6, 1098 (2011)

109. L. Massa, R. Kumar, P. Ravindran, Phys. Fluids 24, 066101 (2012)

110. O. Semeraro, G. Bellani, F. Lundell, Exp. Fluids 5, 1203 (2012)

111. E.J. Candès, Proc. Int. Congr. Math. 3 (2006)

112. Z. Bai, et al., AIAA J. 53, 920 (2014)

113. M.R. Jovanović, P.J. Schmid, J.W. Nichols, Center for Turbulence Res. Annual Research Briefs (2012), pp. 139-152

114. D. Duke, J. Soria, D. Honnery, Experiments in Fluids 52, 529 (2012)

115. A. Wynn, D.S. Pearson, B. Ganapathisubramani, P.J. Goulart, J. Fluid Mech. 733, $473(2013)$

116. B. Koopman, J.V. Neumann, Proc. Natl. Acad. Sci. USA 18, 255 (1932)

117. I. Mezic, Ann. Rev. Fluid Mech. 45, 357 (2013)

118. S. Dawson, N. Schiavone, C. Rowley, D. Williams, 45th AIAA Fluid Dynamics Conference (2015), pp. 1-14

119. J.R. Annoni, J. Nichols, P.J. Seiler, Wind Farm Modeling and Control Using Dynamic Mode Decomposition (American Institute of Aeronautics and Astronautics, 2016)

120. I. Mezić, S. Wiggins, Chaos: An Interdisciplinary J. Nonlinear Sci. 9, 213 (1999)

121. M. Budišić, I. Mezić, Decision and Control, 2009 held jointly with the 2009 28th Chinese Control Conference. CDC/CCC 2009. Proc. 48th IEEE Conf. (IEEE, 2009), p. 3162

122. Y. Lan, I. Mezić, Physica D: Nonlinear Phenomena 242, 42 (2013)

123. S.L. Brunton, J.L. Proctor, J.N. Kutz, Proc. Natl. Acad. Sci. 113, 3932 (2016)

124. I.J. Leontaritis, S.A. Billings, Int. J. Control 41, 303 (1985)

125. S.L. Brunton, J.L.Proctor, J.N. Kutz, [arXiv:1509.03580] (2015)

Open Access This is an Open Access article distributed under the terms of the Creative Commons Attribution License (http://creativecommons.org/licenses/by/4.0), which permits unrestricted use, distribution, and reproduction in any medium, provided the original work is properly cited. 